\title{
EVALUACIÓN DEL TEÑIDO DE FIBRAS NATURALES CON TINTES EXTRAÍDOS DE LA MANZANILLA (Matricaria chamomilla)*
}

\author{
Párraga Melgarejo, Nancy ${ }^{1}$ y Rojas Espinoza, Gonzalo ${ }^{2}$ \\ Facultad de Ciencias Aplicadas. Universidad Nacional del Centro del Perú
}

\begin{abstract}
RESUMEN
Con el propósito de evaluar los colorantes naturales obtenidos de la manzanilla en el teñido de lana de ovino (fibra animal) y el algodón (fibra vegetal), se utilizaron hojas y flores de la especie Matricaria chamomilla $\mathrm{L}$, con el método directo utilizando agua como fuente y como mordiente diferentes proporciones de alumbre (10 y $15 \%)$. Los objetivos fueron: evaluar la tinción de fibra natural animal (lana de ovino) y vegetal (algodón) haciendo uso de colorantes extraídos de la especie Matricaria chamomilla L.; diversificar los usos convencionales que tiene la manzanilla en el campo agroindustrial; darle valor agregado a las fibras naturales como la lana de ovino y el algodón, para mejorar su valor económico. Al final de la investigación se logró determinar que para el caso de la fibra de ovino, los colorantes más estables corresponden a las muestras de hojas a tiempo de ebullición de 60 minutos, mientras que los correspondientes a las flores son un poco menos estables. El teñido en caso del algodón, se hizo, utilizando el $10 \%$ de mordiente y 60 minutos de ebullición; con $15 \%$ de mordiente para tiempos de 40 y 60 minutos de ebullición, observándose diferencias significativas en el color y estabilidad de los mismos. En general, los colores que predominaron en las fibras naturales en estudio fueron los tonos amarillentos.
\end{abstract}

Palabras clave: colorantes naturales, fibras, manzanilla, mordientes, ebullición.

\section{EVALUATION OF NATURAL FIBERS COLORED TINTS EXTRACTED FROM CHAMOMILE (Matricaria chamomilla)}

\begin{abstract}
With the purpose of evaluating the natural dyeings gotten from the chamomile in colorings the ovine wool (animal fiber) and cotton (vegetal fiber), flowers and leaves from the Matricaria chamomilla L. were used, with the direct method using water as source and as mordant different proportions of alum(10 and $15 \%)$. The objectives: to evaluate the dyeing of natural animal fibers (ovine wool) and vegetal (cotton) through the use of extracted coloring from the Matricaria chamomilla L. species; to diversify the conventional uses that the chamomilla L. has in the agro industrial field; to give and added value to these natural fibers as the ovine fiber and cotton, to improve its economical value. At the end of this investigation it was possible to determine that in the case of the lamb fiber, the most stable colorings were the one coming from the leaves at 60 minutes, boiling while those from the flowers are a bit less stable. The coloring in the case of cotton was possible, using $10 \%$ of mordant and 60 minutes of boiling; and $15 \%$ mordant for 40 and 60 minutes boiling, observing significant ire difference in the color and its stability, In general, the predominant colors in natural fibers in studies were yellowish tones.
\end{abstract}

Key Words: natural coloring, fibers, chamomile, mordant, boil.

\footnotetext{
* Este trabajo fue recibido el 20/06/2007, retornado para su revisión el 22/09/2008 y aprobado para publicación el 27/02/2009.

1. E-mail: zpamel@hotmail.com

2. E-mail: gonzalorojas_2006@ hotmail.com
} 


\section{INTRODUCCIÓN}

Los tintes naturales tienen un valor incalculable para las artesanías del país, en virtud de que pueden usarse en el teñido de hilo de algodón y lana, telas, yute, palma, cuero, pieles, plumas, paja, madera, flores naturales y artificiales, jabones, candelas y aserrín. Su valor radica en que los tintes son productos de la naturaleza contrario de los tintes químicos con contenidos de colores, que hacen mucho daño a la naturaleza humana, motivo esgrimido por los europeos para rechazar las artesanías textiles de algunos lugares como de Guatemala (Ginsburg M. ,1998).

La provincia de Tarma cuenta con recursos vegetales que no son explotados racionalmente por desconocimiento de formas de dotarle de valor agregado como es el caso de algunas hierbas aromáticas de la zona.

Existe la necesidad de realizar investigaciones en torno a lo referido, en este caso particular se quiso averiguar respecto a la Matricaria chamomilla L (manzanilla) como tiente natural para el teñido de fibras naturales, en el marco del campo agroindustrial y en un intento por querer contribuir a mejorar la calidad de vida de los pobladores de esta parte del país.

Se tuvo en cuenta los siguientes objetivos:

General:

Evaluar la tinción de fibras naturales animal y vegetal haciendo uso de colorantes extraídos de la especie Matricaria chamomilla L.

\section{Específicos:}

- Diversificar los usos convencionales que tiene la manzanilla en el campo agroindustrial.

- Darle valor agregado a las fibras naturales como la lana de ovino y el algodón, para mejorar su valor económico.

La tecnología utilizada en esta investigación puede ser transferida a los pequeños y medianos empresarios agroindustriales con éxito debido a que es totalmente asequible a ellos contribuyendo con la mejora de la actividad económica del poblador rural de nuestra provincia.

\section{MATERIAL Y MÉTODOS}

\section{Materiales y equipos utilizados}

Lana de ovino, algodón, tallos, hojas de manzanilla, alcohol etílico, agua destilada, navajas, tijeras de podar, recipientes, agitadores, bolsas de polietileno, telas, bandejas, matraces, fiolas, tubos de ensayo, pipetas, vasos de precipitación, libreta de apuntes, cámara fotográfica, balanza de precisión, cronómetro, equipo Soxhlet, estufa, termómetro.

El método usado fue el experimental que consistió en: Seleccionar, identificar y obtener las hojas y flores a trabajar de la Matricaria chamomilla L.

\section{a) Identificación y obtención de la Matricaria} chamomilla para obtener los tintes naturales: Las partes que fueron evaluadas de la Matricaria chamomilla L. (hojas y flores), procedieron de cultivos de la provincia de Tarma, especialmente de las zonas de Picoy y Huanuquillo.

b) Extracción del tinte: Para la extracción de pigmentos en la Matricaria chamomilla L. las partes utilizadas fueron hojas y flores; las cuales fueron frescas. Las plantas que se usaron se hirvieron con agua en un recipiente donde también se introdujeron las fibras. El tiempo aproximado para la tinción es de un mínimo de 30 minutos, durante el cual se agitó constantemente, para favorecer una difusión homogénea del tinte en la fibra (Portillo M. y Vigueras C., 1995).

\section{c) Proceso de la tinción:}

Método directo. Que consiste en introducir la fibra directamente al tinte. Para el trabajo de investigación se utilizó 10 y $15 \%$ de mordiente (alumbre) y a tiempos de ebullición de 40 y 60 minutos por cada 50 gramos de fibra natural que constituyeron las muestras, tanto para la lana de ovino como para el algodón.

Mordentado. Se introdujo la fibra sin teñir en agua tibia que contenía el mordiente en cantidad de $100 \mathrm{ml}$. para que cubra la fibra. Se calentó a un punto de ebullición por un lapso de tiempo de 40 ó 60 minutos de acuerdo a las muestras, agitando constantemente. Antes de proceder a la preparación del baño de mordentado, se examinó la cantidad de fibra (para todos los casos $50 \mathrm{~g}$.) y alumbre $5 \mathrm{~g}$. ó 7,5g. 
dependiendo de los tratamientos. Antes de mordentar se humedeció la fibra completamente. Además, las fibras que se tiñeron estuvieron limpias de impurezas para que puedan retener mejor el colorante.

d) Evaluación de la tinción de fibras naturales: Se realizó tomando en cuenta el porcentaje de mordiente, y los tiempos respectivos en cada muestra trabajada, las observaciones fueron tomadas en cuenta de acuerdo a la intensidad y la estabilidad de color que presentaron; la lana de ovino, se torna una fibra un poco más difícil para el teñido debido a que la queratina presente en su estructura (Arosteguy, R. 1992).

e) Calidad de teñido: fueron determinados de acuerdo a las evaluaciones que se realizaron por observación directa del color de la fibra, de acuerdo a la intensidad que éstas presentaban.

\section{RESULTADOS}

a) Identificación y obtención de la Matricaria chamomilla para obtener los tintes naturales. EI estudio realizado para el diagnóstico de la población de manzanilla en la provincia de Tarma, se llevó a cabo mediante observación directa además de referencias de las instituciones relacionadas a este campo (PRONAMACHS, MINAG). Habiendo encontrado la información siguiente:

Población aproximada de Matricaria chamomilla en la Provincia de Tarma es de 13 hectáreas. (Agencia Agraria Tarma, 2007). La mayor cantidad de éstas están localizadas en Picoy, Huanuquillo, Ingenio, Pomachaca, Huaylahuichán y Sacsamarca.

La mayoría de estos cultivos son para venta directa en fresco y otro porcentaje considerable $40 \%$ para la deshidratación y comercialización a la ciudad de Lima para hierbas aromáticas en forma de filtros (Ministerio de Agricultura-Agencia Agraria Tarma, 2007).

\section{b) Extracción del tinte:}

Se obtuvieron los colorantes de la Matricaria chamomilla, de dos órganos de la planta: hojas y flores. Habiéndose trabajado para cada uno de los casos, la extracción directa.
Cuadro 1. Porcentaje y cantidad de mordiente utilizado para el teñido.

\begin{tabular}{ccc}
\hline $\begin{array}{c}\text { Lana/algodón } \\
50 \mathrm{~g} .\end{array}$ & Mordiente \% & $\begin{array}{c}\text { Equivalencia } \\
\text { (gramos) }\end{array}$ \\
\hline $50 \mathrm{~g}$. & Alumbre $15 \%$ & 7,5 \\
& Alumbre $10 \%$ & 5,0 \\
\hline
\end{tabular}

El mordiente alumbre se disuelve en poca agua caliente y luego se agrega agua fría hasta $100 \mathrm{ml}$. Para cubrir las madejas que se van a teñir.

\section{Proceso para teñir la fibra vegetal: Los tiempos utilizados}

Para lograr un buen tinturado, se debe tener muy en cuenta las cantidades de lana, mordiente, planta, agua y tiempo de ebullición.

Cuadro 2. Porcentaje de mordiente y tiempos de ebullición en el teñido.

\begin{tabular}{ccc}
\hline Lana/algodón & Mordiente $\%$ & $\begin{array}{c}\text { Tiempo de } \\
\text { Ebullición }\end{array}$ \\
\hline $50 \mathrm{~g}$. & Alumbre $15 \%$ & 40 minutos \\
$50 \mathrm{~g}$. & Alumbre $10 \%$ & 40 minutos \\
$50 \mathrm{~g}$. & Alumbre $15 \%$ & 60 minutos \\
$50 \mathrm{~g}$. & Alumbre $10 \%$ & 60 minutos \\
\hline
\end{tabular}

La cantidad de hojas y flores para cada una de las muestras fue de 50 gramos.

La madeja húmeda se introdujo en el agua con mordiente y se puso a hervir a fuego lento por el tiempo referido en el cuadro 2. Luego se retiró para enjuagar, se utilizó agua tibia $\left(23^{\circ} \mathrm{C}\right)$.

La mayoría de los tintes naturales requieren de ciertos fijadores o asistentes para poder teñir, estas substancias son denominadas mordientes, mismas que pueden ser de origen natural o químico, las cuales facilitan la fijación del tinte a la fibra, en determinados tiempos además funcionan como elementos de uniformidad y brillo de color (Portillo M. y Vigueras C. , 1995).

Cuadro 3. Lana de ovino (50 g.) con $10 \%$ de mordiente y 60 minutos de ebullición.

\begin{tabular}{ccc}
\hline $\begin{array}{c}\text { Órgano del } \\
\text { vegetal }\end{array}$ & Color & Estabilidad \\
\hline Hojas & Verde amarillento & Regular \\
Flores & Amarillento & Buena \\
\hline
\end{tabular}


Cuadro 4. Algodón $(50$ g.) con $15 \%$ de mordiente y 40 minutos de ebullición.

\begin{tabular}{ccc}
\hline $\begin{array}{c}\text { Órgano del } \\
\text { vegetal }\end{array}$ & Color & Estabilidad \\
\hline Hojas & Verde amarillento & Buena \\
Flores & Amarillento & Buena \\
\hline
\end{tabular}

Cuadro 5. Algodón (50 g.) con $15 \%$ de mordiente y 60 minutos de ebullición.

\begin{tabular}{ccc}
\hline $\begin{array}{c}\text { Órgano del } \\
\text { vegetal }\end{array}$ & Color & Estabilidad \\
\hline Hojas & Verde amarillento & Buena \\
Flores & amarillento (intenso) & Buena \\
\hline
\end{tabular}

Los resultados obtenidos en la investigación, nos indican que los colorantes más estables corresponden a las muestras de flores a tiempo de ebullición de 60 minutos, mientras que los correspondientes a las hojas son un poco menos estables para el caso de la lana de ovino, puesto que ésta, se torna una fibra un poco más difícil para el teñido debido a la queratina presente en su estructura (Arosteguy, R. 1992).

Cabe remarcar, que en el caso del algodón esta estabilidad es más notoria, incluso con 40 minutos de ebullición. Ya que el algodón, es considerado como una fibra suave, es la planta textil más importante (Puente J., 2001). Cada una de estas pruebas fueron repetidas en tres oportunidades, se hicieron uso de las hojas y flores de la manzanilla recién obtenidas (frescas), obteniéndose de ellas buenos resultados, en cuanto a color y estabilidad; tanto en la lana de ovino como en el algodón.

\section{c) Proceso de tinción:}

El proceso de tinción utilizado en la investigación fue el artesanal, que se aplicó con la solución tintórea líquida obtenida de la Matricaria chamomilla por ebullición (método directo). Para que el color penetre en las fibras y se fije, se efectúo con el uso de mordiente (fijador). La mayoría de los tintes naturales requieren de ciertos fijadores o asistentes para poder teñir, estas substancias son denominadas mordientes, mismas que pueden ser de origen natural o químico, las cuales facilitan la fijación del tinte a la fibra, en determinados tiempos además funcionan como elementos de uniformidad y brillo de color (Portillo M. y Vigueras C. ,1995).

Es necesario que las fibras estén mojadas por completo antes de teñirse; asimismo, que los recipientes donde se mordente o tiña deberán ser de material no reactivo preferentemente (Puente J., 2001). Además, la lana que se van a teñir deberá estar limpia de impurezas para que pueda retener mejor el colorante, pues elementos como la grasa impiden la retención del mismo. Se recomienda lavarlas con agua tibia y jabón en cantidades suficientes para un lavado normal. Para que la lana no se enrede y tome una coloración uniforme al momento de teñirla, es necesario elaborar pequeñas madejas, esto con la finalidad de facilitar su manejo.

\section{DISCUSIÓN}

Los tintes o colorantes obtenidos en cada uno de los casos fueron utilizados para el teñido de las fibras naturales. Los que se realizaron en dos grupos: 1) Lana de ovino. 2) Algodón. Como se puede notar, la lana de ovino, se torna una fibra un poco más difícil para el teñido debido a que la queratina presente en su estructura lo dificulta; como refiere Ginsburg M. (1998) “la capa cortical constituye el $90 \%$ de la fibra de la lana de ovino está formada por células alargadas fusiformes que contienen queratina. La queratina constituye un polímero natural que presenta la composición química elemental: $51 \%$ de carbono, $17 \%$ de nitrógeno, $22 \%$ de oxígeno, $7 \%$ de hidrógeno y de $3 \%$ de azufre. Protege el cuerpo del medio externo y es por ello insoluble en agua" (Ginsburg M. 1998) pero, denotándose que el teñido es más firme y de color más intenso.

El teñido en caso del algodón, se dio, incluso habiendo utilizado el $10 \%$ de mordiente y 60 minutos de ebullición; como con $15 \%$ de mordiente para tiempos de 40 y 60 minutos de ebullición, no observándose diferencias significativas en el color y estabilidad de los mismos. (Como se pueden observar las muestras, anexos). Corroborándose, que el algodón, es considerado como una fibra suave, es la planta textil más importante (Puente J., 2001). En general, los colores que predominaron fueron los tonos amarillentos. "El color amarillo es el más común de obtenerse a partir de las plantas (Portillo M. y Vigueras C., 1995). 


\section{CONCLUSIONES}

Los resultados obtenidos en la investigación denotan lo siguiente:

- Para el caso de la fibra de ovino, los colorantes más estables corresponden a las muestras de flores a tiempo de ebullición de 60 minutos, mientras que los correspondientes a las hojas son un poco menos estables.

- El teñido en caso del algodón, se dio, utilizando el $10 \%$ de mordiente y 60 minutos de ebullición; como con $15 \%$ de mordiente para tiempos de 40 y 60 minutos de ebullición, no observándose diferencias significativas en el color y estabilidad de los mismos.

- Se encontró que en la lana de ovino, el teñido es más firme y de color más intenso que en el algodón.

- En general, los colores que predominaron en las fibras naturales en estudio fueron los tonos amarillentos.

- En nuestro medio si es factible el dotarle valor agregado a las fibras naturales tanto de origen animal como vegetal y aprovechar en forma óptima y diversificada lo recursos naturales de nuestro entorno.

\section{LITERATURA CITADA}

Arosteguy, R. 1992. Lanares cuaderno de actualización técnica. 1a ed., Consorcio Regional de Experimentación Agrícola. Argentina. p 203.

Ginsburg, M. 1998. La historia de los textiles. Editorial. LIBSA. Madrid, España. P.53.

Malo, G. C. 2000. Diseño y artesanía. Centro lberoamericano de Artesanías y Artes Populares. Cuenca, Ecuador. p.120.

Monella, E. S. 2001. Aprovechamiento integral de especies vegetales aromáticas y medicinales. SIT INGENIERÍA S.R.L. Santa Fe. Argentina. p. 120 .

Munóz, F. 1998. Plantas medicinales y aromáticas: estudio, cultivo y procesado. Editorial MundiPrensa. Madrid. España. p. 260.

Nencataco, A. 1996. Manual de tintes naturales. Corporación de Artes Textiles. Artesanías de Colombia-OEA. Bogotá.

Portillo, M. y Vigueras, C. 1995. Teñido con colorantes naturales. Traducido del Inglés por Mario Paz. Editorial Acribia. Zaragoza-España. p. 275.

Puente, J. P. 2001. Fundamentos científicos y aplicados de la tintura de materiales textiles. Universidad Politécnica de Barcelona. ETSIT Terrassa. España. p. 86.

Roo, A. y Córdova, C. 1991. Manual de tintes de origen natural para lana. Ediciones del Serval. Madrid. p. 95. 